\title{
Boundary feedback stabilization of hydraulic jumps
}

\author{
Georges Bastin ${ }^{\mathrm{a}}$, Jean-Michel Coron ${ }^{\mathrm{b}}$, Amaury Hayat ${ }^{\mathrm{b}}$, and Peipei Shang ${ }^{\mathrm{c}}$ \\ ${ }^{a}$ Department of Mathematical Engineering, ICTEAM, University of Louvain, Louvain-La-Neuve, Belgium. \\ ${ }^{\mathrm{b}}$ Sorbonne Université, Université Paris-Diderot SPC, CNRS, INRIA, Laboratoire Jacques-Louis Lions, \\ équipe Cage, Paris, France. \\ ${ }^{\mathrm{c}}$ School of Mathematical Sciences, Tongji University, Shanghai, China.
}

\begin{abstract}
In an open channel, a hydraulic jump is an abrupt transition between a torrential (supercritical) flow and a fluvial (subcritical) flow. In this article hydraulic jumps are represented by discontinuous shock solutions of hyperbolic Saint-Venant equations. Using a Lyapunov approach, we prove that we can stabilize the state of the system in $H^{2}$-norm as well as the hydraulic jump location, with simple feedback boundary controls and an arbitrary decay rate, by appropriately choosing the gains of the feedback boundary controls.
\end{abstract}

Keywords. Saint-Venant equations, boundary feedback controls, hydraulic jump.

AMS Subject Classification. 93D15, 35L40, 35L67.

\section{Introduction and main result}

Nonlinear hyperbolic equations are well-known to give rise to discontinuities in finite time that are physically meaningful. Hydraulic jump is one of the most known example. A hydraulic jump is a phenomenon that frequently occurs in open channel flow, such as rivers and spillways. It describes a transition between a torrential (or supercritical) regime and a fluvial (or subcritical) regime, i.e., an abrupt transition between a fast flow and a slow flow with a higher height. As a consequence, a part of the initial kinetic energy of the flow is converted into an increase in potential energy, while some energy is irreversibly lost through turbulence and heat. This phenomenon can be seen not only in rivers and spillways but also in air flows of the atmosphere. This is for instance believed to explain the phenomenon of "Morning Glory cloud" [7] and may be at the origin of some gliders' crashes [18. Hydraulic jumps are important not only because they occur naturally but also because they are sometimes engineered on purpose and are very useful in hydraulic applications to dissipate energy in water and prevent in this way the erosion of the streambed or damages on hydraulic installations [16. However, when studying the flow equations, the stabilization of hydraulic jumps is seldom considered and almost all the studies focus on the stabilization of the dynamics of the fluvial regime 1, 2, 3, 4, 9, 11, 14, 19. In this paper, we explicitly address the issue of the stabilization of a hydraulic jump represented by a discontinuous shock solution of the flow equations, switching from the torrential regime to the fluvial regime. In other words, the two eigenvalues of the hyperbolic system modeling the shallow water are both positive in the torrential regime and one of them changes sign and switches to a negative value in the fluvial regime. Our goal is to achieve the stability of the channel with a general class of local feedback controls at the boundary. Fundamentally, the stabilization of shock steady states for hyperbolic systems, while being very interesting, has rarely been studied. One can refer to [5] and 21] for the scalar case and to our knowledge, no such result exists for systems.

By a Lyapunov approach we prove the exponential $H^{2}$-stability of the steady state, with an arbitrary decay rate and with an exact exponential stabilization of the desired location of the hydraulic jump.

We consider a channel with a rectangular cross section with constant width, which is taken to be 1 without loss of generality. We denote by $Q(t, x)$ the flux and $H(t, x)$ the water depth, where $t$ and $x$ are, respectively, the time and space independent variables as usual. As the channel has a finite length 
$L>0$, the spatial domain is bounded and noted $[0, L]$. The Saint-Venant model which, neglecting friction, consists in a continuity equation and an equilibrium of forces, is written as

$$
\begin{aligned}
& \partial_{t} H+\partial_{x} Q=0, \\
& \partial_{t} Q+\partial_{x}\left(\frac{g H^{2}}{2}+\frac{Q^{2}}{H}\right)=0 .
\end{aligned}
$$

We are interested with solution trajectories $(H(t, x), Q(t, x))^{\top}$ that may have a jump discontinuity at some point $x_{s}(t) \in(0, L)$ and are classical otherwise. Thus, in order to close the system, we need a relationship between $Q$ and $H$ before and after this jump. From the Rankine-Hugoniot condition applied to (1), two quantities are conserved through the jump in the jump's referential: the flux $Q$ and the momentum $g H^{2} / 2+Q^{2} / H$. This gives the following relationships at the jump $x_{s}(t)$ :

$$
\begin{aligned}
& {[Q]_{-}^{+}=\dot{x}_{s}[H]_{-}^{+},} \\
& {[Q]_{-}^{+} \dot{x}_{s}=\left[\frac{Q^{2}}{H}+\frac{1}{2} g H^{2}\right]_{-}^{+},}
\end{aligned}
$$

where, as usual, $\dot{x}_{s}$ denotes the time derivative of $x_{s}$, i.e., the speed of the jump. These relationships can be reformulated as:

$$
\begin{gathered}
\dot{x}_{s}=\frac{[Q]_{-}^{+}}{[H]_{-}^{+}} \\
\text {and }\left([Q]_{-}^{+}\right)^{2}=[H]_{-}^{+}\left[\frac{Q^{2}}{H}+\frac{1}{2} g H^{2}\right]_{-}^{+},
\end{gathered}
$$

where we define for any bounded function $f$ in a neighbourhood of $x_{s}$ : $[f]_{-}^{+}=f\left(x_{s}^{+}(t)\right)-f\left(x_{s}^{-}(t)\right)$. This relation (4) can be regarded as the generalisation for non-stationary states of the well-known Bélanger equation (8) below.

Our goal is to stabilize the steady states of the system (1), (3) and (4) where a (single) hydraulic jump occurs, meaning that the flow switches from the torrential regime to the fluvial regime with a discontinuity in height. Therefore, such steady states $\left(\left(H^{*}, Q^{*}\right)^{\top}, x_{s}^{*}\right)$ satisfy the following conditions:

1. $Q^{*}$ is constant and positive, $x_{s}^{*} \in(0, L)$ and

$$
H^{*}= \begin{cases}H_{1}^{*}, & x \in\left[0, x_{s}^{*}\right), \\ H_{2}^{*}, & x \in\left(x_{s}^{*}, L\right]\end{cases}
$$

where $H_{1}^{*}, H_{2}^{*}$ are positive constants.

2. The steady state flow is in the torrential regime before the jump and in the fluvial regime after the jump. This means that in the torrential regime the two system eigenvalues are positive,

$$
\lambda_{1}=\frac{Q^{*}}{H_{1}^{*}}-\sqrt{g H_{1}^{*}}>0, \quad \lambda_{2}=\frac{Q^{*}}{H_{1}^{*}}+\sqrt{g H_{1}^{*}}>0, \quad \text { for } x \in\left[0, x_{s}^{*}\right),
$$

while there is one positive and one negative eigenvalue in the fluvial regime [2,

$$
-\lambda_{3}=\frac{Q^{*}}{H_{2}^{*}}-\sqrt{g H_{2}^{*}}<0, \quad \lambda_{4}=\frac{Q^{*}}{H_{2}^{*}}+\sqrt{g H_{2}^{*}}>0, \quad \text { for } x \in\left(x_{s}^{*}, L\right] .
$$

In particular this implies that $H_{1}^{*}<H_{2}^{*}$.

3. Furthermore, the Rankine-Hugoniot conditions applied to (1) in the stationary case are equivalent to the following well-known Bélanger equation [6]

$$
\frac{H_{2}^{*}}{H_{1}^{*}}=\frac{-1+\sqrt{1+8 \frac{\left(Q^{*}\right)^{2}}{g\left(H_{1}^{*}\right)^{3}}}}{2} .
$$




\section{Physical remarks:}

- The switch from the torrential regime to the fluvial regime corresponds to a transition (shock) between a state where the system (11) has two positive eigenvalues and a state where the system has one positive and one negative eigenvalue. As we will see later (from Theorem 1.1 together with (6) and (7)), this transition (shock) induces a discontinuity not only for the eigenvalue that changes sign but also for the eigenvalue that keeps the same sign. More precisely, if we denote by $\lambda_{s}$ the eigenvalue that changes sign, then $\lambda_{s}\left(x_{s}^{-}(t)\right)>0>\lambda_{s}\left(x_{s}^{+}(t)\right)$ for all $t>0$. And if we denote by $\lambda_{c}$ the eigenvalue that does not change sign, then $\lambda_{c}\left(x_{s}^{-}(t)\right) \neq \lambda_{c}\left(x_{s}^{+}(t)\right)$ for all $t>0$. We point out that smooth transitions could happen around critical equilibria or when source terms are considered (see [10], 15]). Such smooth transitions are also related to coupling conditions for networks for the transition from supersonic to subsonic fluid states, such as natural gas pipeline transportation systems that have been analyzed in [13.

- Note that when the solutions are classical, the formulation (1) of the Saint-Venant equations with the level $H$ and the flux $Q$ as state variables is equivalent to the alternative formulation with the level $H$ and the velocity $Q / H$ that is obtained by replacing the equilibrium of forces by an energy equation and is used for instance in $2,3,17$. When the solutions are not classical however, the two formulations are not equivalent anymore and this can be seen by looking at the stationary states: the formulation (1) in level and flux is compatible with shock and discontinuity of $H^{*}(x)$ while the version with the energy equation is not. This is logical as there is a pointwise loss of energy in the hydraulic jump, which implies that the energy conservation does not hold anymore.

- From (3), the location of the shock $x_{s}$ may be moving around its initial location and potentially all along the channel. This can be seen in practical phenomena such as tidal bores. The main challenge of this work is to also stabilize this location when stabilizing the state of the system. This is not obvious as one can see that for given heights and flux $\left(H_{1}^{*}, H_{2}^{*}, Q^{*}\right)$ satisfying (6)-(8), any shock location $x_{s}^{*} \in[0, L]$ induces an admissible steady state $\left(\left(H^{*}, Q^{*}\right)^{\top}, x_{s}^{*}\right)$, where $H^{*}$ is given by (5). Thus the steady states are not isolated and therefore not asymptotically stable in open loop. Indeed, any small perturbation on $x_{s}^{*}$ corresponds to another steady state with the same heights and flux at the two ends.

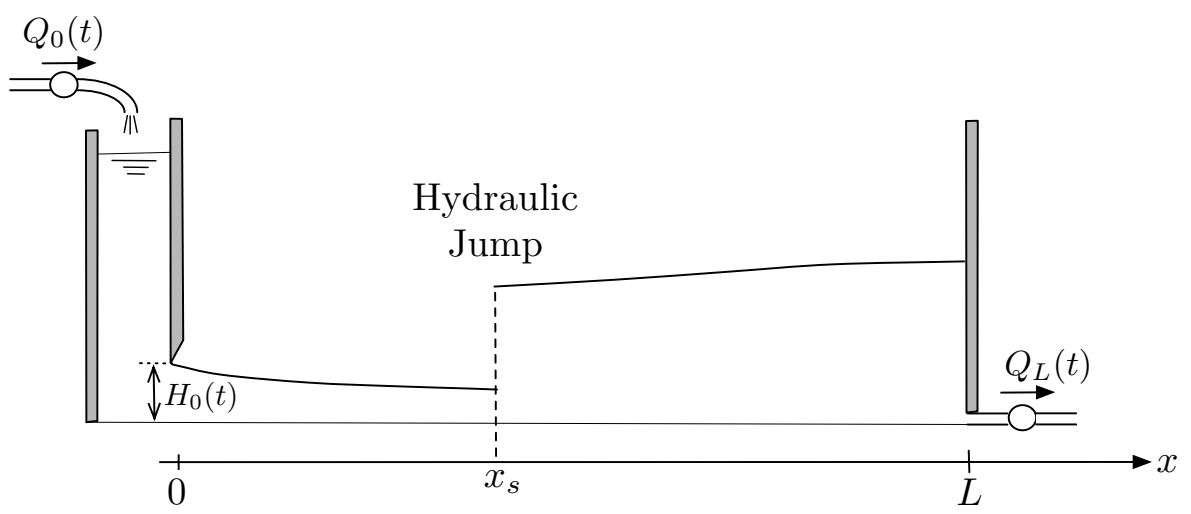

Figure 1: Open channel with a hydraulic jump and three control devices : the gate opening $H_{0}(t)$ and the inflow $Q_{0}(t)$ and outflow $Q_{L}(t)$ which are driven by two pumps.

As illustrated in Figure 1, let us consider a channel which is equipped with devices allowing a feedback control on $H(t, 0)=H_{0}(t), Q(t, L)=Q_{L}(t)$ and $Q(t, 0) \approx Q_{0}(t)$ (quasi-steady state approximation). Let the set point for the control be a steady state $\left(\left(H^{*}, Q^{*}\right)^{\top}, x_{s}^{*}\right)$ defined as previously by (5)-(8). We assume that static boundary feedback control laws are selected so that the boundary 
conditions can be written in the following general form:

$$
\left(\begin{array}{c}
H(t, 0)-H_{1}^{*} \\
Q(t, 0)-Q^{*} \\
Q(t, L)-Q^{*}
\end{array}\right)=G\left(\begin{array}{c}
Q\left(t, x_{s}^{-}\right)-Q^{*} \\
Q\left(t, x_{s}^{+}\right)-Q^{*} \\
H\left(t, x_{s}^{-}\right)-H_{1}^{*} \\
x_{s}-x_{s}^{*}
\end{array}\right)-\left(\begin{array}{c}
0 \\
0 \\
G_{4}\left(H(t, L)-H_{2}^{*}\right)
\end{array}\right)
$$

where $G=\left(G_{1}, G_{2}, G_{3}\right)^{\top}: \mathbb{R}^{4} \rightarrow \mathbb{R}^{3}$ and $G_{4}: \mathbb{R} \rightarrow \mathbb{R}$ are of class $C^{2}$ and satisfy

$$
G(\mathbf{0})=\mathbf{0}, \quad G_{4}(0)=0, \quad G_{4}^{\prime}(0)=-\lambda_{4} .
$$

Obviously, by (5), the steady state $\left(\left(H^{*}, Q^{*}\right)^{\top}, x_{s}^{*}\right)$ satisfies the boundary conditions $(9)$, as $H^{*}(0)=$ $H_{1}^{*}$ and $H^{*}(L)=H_{2}^{*}$. Note that this boundary feedback is quite simple to implement as it only requires a pointwise measure of $H(t, L), x_{s}(t), H\left(t, x_{s}^{-}\right), Q\left(t, x_{s}^{+}\right)$and $Q\left(t, x_{s}^{-}\right)$.

In order to state the main stability result of this article, we first introduce the following notations:

$$
\begin{aligned}
& D(x, \gamma)=\operatorname{diag}\left(\frac{s_{i}\left(1-s_{i} \frac{\lambda_{i}}{\lambda_{4}}\right)}{b_{i}} e^{\frac{\gamma}{x_{i} \lambda_{i}}\left(x_{s}^{*}-x\right)}, i \in\{1,2,3\}\right), \\
& \widetilde{D}(\gamma)=\operatorname{diag}\left(\left(\sum_{j=1}^{3} e^{\frac{\gamma x_{s}^{*}}{x_{i} \lambda_{i}}-\frac{\gamma x_{s}^{*}}{x_{j} \lambda_{j}}}\right)\left(1-s_{i} \frac{\lambda_{i}}{\lambda_{4}}\right)^{2}, i \in\{1,2,3\}\right), \\
& K=\left(\begin{array}{ccc}
\frac{\lambda_{2} \lambda_{1}}{\lambda_{2}-\lambda_{1}} & -\frac{\lambda_{1}}{\lambda_{2}-\lambda_{1}} & 0 \\
\frac{\lambda_{2} \lambda_{1}}{\lambda_{1}-\lambda_{2}} & -\frac{\lambda_{2}}{\lambda_{1}-\lambda_{2}} & 0 \\
0 & 0 & \frac{\lambda_{3}}{\lambda_{3}+\lambda_{4}}
\end{array}\right) G^{\prime}(\mathbf{0})\left(\begin{array}{ccc}
1 & 1 & 0 \\
\frac{\lambda_{1}}{\lambda_{4}} & \frac{\lambda_{2}}{\lambda_{4}} & 1+\frac{\lambda_{3}}{\lambda_{4}} \\
\frac{1}{\lambda_{1}} & \frac{1}{\lambda_{2}} & 0 \\
0 & 0 & 0
\end{array}\right) \text {, } \\
& d=\frac{1}{H_{1}^{*}-H_{2}^{*}}, \\
& \left(\begin{array}{l}
b_{1} \\
b_{2} \\
b_{3}
\end{array}\right)=\left(\begin{array}{ccc}
\frac{\lambda_{2} \lambda_{1}}{\lambda_{2}-\lambda_{1}} & -\frac{\lambda_{1}}{\lambda_{2}-\lambda_{1}} & 0 \\
\frac{\lambda_{2} \lambda_{1}}{\lambda_{1}-\lambda_{2}} & -\frac{\lambda_{2}}{\lambda_{1}-\lambda_{2}} & 0 \\
0 & 0 & \frac{\lambda_{3}}{\lambda_{3}+\lambda_{4}}
\end{array}\right) G^{\prime}(\mathbf{0})\left(\begin{array}{l}
0 \\
0 \\
0 \\
1
\end{array}\right)
\end{aligned}
$$

with $s_{1}=s_{2}=1, s_{3}=-1, x_{1}=x_{2}=1, x_{3}=x_{s}^{*} /\left(L-x_{s}^{*}\right)$ and $x_{4}=x_{s}^{*} /\left(x_{s}^{*}-L\right)$.

We consider the following initial condition

$$
H(0, x)=H_{0}(x), \quad Q(0, x)=Q_{0}(x), \quad x_{s}(0)=x_{s, 0}
$$

where $x_{s, 0} \in(0, L)$ and $\left(H_{0}(x), Q_{0}(x)\right)^{\top} \in H^{2}\left(\left(0, x_{s, 0}\right) ; \mathbb{R}^{2}\right) \cap H^{2}\left(\left(x_{s, 0}, L\right) ; \mathbb{R}^{2}\right)$. We assume that the initial condition satisfies the first order compatibility conditions derived from (9), (see [2] for a proper definition of the first order compatibility condition which is omitted here for the sake of simplicity). Now, we give the following definition:

Definition 1.1. The steady state $\left(\left(H^{*}, Q^{*}\right)^{\top}, x_{s}^{*}\right)$ is locally exponentially stable for the $H^{2}$-norm with decay rate $\gamma$, if there exist $\delta^{*}>0$ and $C^{*}>0$ such that for any initial data $\left(H_{0}(x), Q_{0}(x)\right)^{\top} \in$ $H^{2}\left(\left(0, x_{s, 0}\right) ; \mathbb{R}^{2}\right) \cap H^{2}\left(\left(x_{s, 0}, L\right) ; \mathbb{R}^{2}\right)$ and $x_{s, 0} \in(0, L)$ satisfying

$$
\begin{aligned}
& \left|\left(H_{0}-H_{1}^{*}, Q_{0}-Q^{*}\right)^{\top}\right|_{H^{2}\left(\left(0, x_{s, 0}\right) ; \mathbb{R}^{2}\right)}+\left|\left(H_{0}-H_{2}^{*}, Q_{0}-Q^{*}\right)^{\top}\right|_{H^{2}\left(\left(x_{s, 0}, L\right) ; \mathbb{R}^{2}\right)} \leq \delta^{*}, \\
& \left|x_{s, 0}-x_{s}^{*}\right| \leq \delta^{*}
\end{aligned}
$$

and the corresponding first order compatibility conditions derived from (9), and for any $T>0$, the system (1), (3), (4), (9) and $(12)$ has a unique solution $(H, Q)^{\top} \in C^{0}\left([0, T] ; H^{2}\left(\left(0, x_{s}(t)\right) ; \mathbb{R}^{2}\right) \cap\right.$ 


$$
\begin{aligned}
& \left.H^{2}\left(\left(x_{s}(t), L\right) ; \mathbb{R}^{2}\right)\right) \text { and } x_{s} \in C^{1}([0, T]) \text { and } \\
& \qquad \begin{aligned}
\mid\left(H(t, \cdot)-H_{1}^{*}, Q(t, \cdot)-\right. & \left.Q^{*}\right)\left.^{\top}\right|_{H^{2}\left(\left(0, x_{s}(t)\right) ; \mathbb{R}^{2}\right)} \\
& +\left|\left(H(t, \cdot)-H_{2}^{*}, Q(t, \cdot)-Q^{*}\right)^{\top}\right|_{H^{2}\left(\left(x_{s}(t), L\right) ; \mathbb{R}^{2}\right)}+\left|x_{s}(t)-x_{s}^{*}\right| \\
\leq C^{*} e^{-\gamma t} & \left(\left|\left(H_{0}-H_{1}^{*}, Q_{0}-Q^{*}\right)^{\top}\right|_{H^{2}\left(\left(0, x_{s, 0}\right) ; \mathbb{R}^{2}\right)}\right. \\
& \left.+\left|\left(H_{0}-H_{2}^{*}, Q_{0}-Q^{*}\right)^{\top}\right|_{H^{2}\left(\left(x_{s, 0}, L\right) ; \mathbb{R}^{2}\right)}+\left|x_{s, 0}-x_{s}^{*}\right|\right), \quad \forall t \in[0, T) .
\end{aligned}
\end{aligned}
$$

Remark 1. A function $f$ in $C^{0}\left([0, T] ; H^{2}\left(\left(0, x_{s}(t)\right) ; \mathbb{R}^{2}\right) \cap H^{2}\left(\left(x_{s}(t), L\right) ; \mathbb{R}^{2}\right)\right)$ is a function $f$ in $C^{0}\left([0, T] ; L^{2}\left((0, L) ; \mathbb{R}^{2}\right)\right)$ such that, if one defines

$$
\begin{gathered}
f_{1}(t, x):=f\left(t, x_{s}(t) x\right), t \in(0, T), x \in(0,1), \\
f_{2}(t, x):=f\left(t, L+\left(x_{s}(t)-L\right) x\right), t \in(0, T), x \in(0,1),
\end{gathered}
$$

then $f_{1}$ and $f_{2}$ are both in $C^{0}\left([0, T] ; H^{2}\left((0,1) ; \mathbb{R}^{2}\right)\right)$. The transformation $f \rightarrow\left(f_{1}, f_{2}\right)$ enables us to reduce the problem to a time-invariant domain and to define the stability of a function $f \in$ $C^{0}\left([0, T] ; H^{2}\left(\left(0, x_{s}(t)\right) ; \mathbb{R}^{2}\right) \cap H^{2}\left(\left(x_{s}(t), L\right) ; \mathbb{R}^{2}\right)\right)$, a function that is piecewise $H^{2}$ with a discontinuity that is potentially moving. This transformation will also be used later on in the analysis of the problem (see 23 below).

Based on Definition 1.1, we have the following theorem.

Theorem 1.1. For any given steady state $\left(\left(H^{*}, Q^{*}\right)^{\top}, x_{s}^{*}\right)$ of the system (1) satisfying (5)-(8) and the boundary conditions (9), for any $\gamma>0$,

if for $i=1,2,3$

$$
\begin{aligned}
& b_{i} \in\left(\frac{-\gamma e^{-\frac{\gamma}{x_{i} \lambda_{i}} x_{s}^{*}}}{3 d s_{i}\left(1-s_{i} \frac{\lambda_{i}}{\lambda_{4}}\right)\left(1-e^{-\frac{\gamma}{x_{i} \lambda_{i}} x_{s}^{*}}\right)}, \frac{-\gamma e^{-\frac{\gamma}{x_{i} \lambda_{i}} x_{s}^{*}}}{3 d s_{i}\left(1-s_{i} \frac{\lambda_{i}}{\lambda_{4}}\right)}\right), \quad \text { if } \quad s_{i}\left(1-s_{i} \frac{\lambda_{i}}{\lambda_{4}}\right)<0, \\
& b_{i} \in\left(\frac{-\gamma e^{-\frac{\gamma}{x_{i} \lambda_{i}} x_{s}^{*}}}{3 d s_{i}\left(1-s_{i} \frac{\lambda_{i}}{\lambda_{4}}\right)}, \frac{-\gamma x_{i} e^{-\frac{\gamma}{x_{i} \lambda_{i}} x_{s}^{*}}}{3 d s_{i}\left(1-s_{i} \frac{\lambda_{i}}{\lambda_{4}}\right)\left(1-e^{-\frac{\gamma}{x_{i} \lambda_{i}} x_{s}^{*}}\right)}\right), \quad \text { if } \quad s_{i}\left(1-s_{i} \frac{\lambda_{i}}{\lambda_{4}}\right)>0,
\end{aligned}
$$

and if the matrix

$$
D\left(x_{s}^{*}, \gamma\right)-K^{\top} D(0, \gamma) K-\left(\sum_{k=1}^{3} \frac{2 d^{2}}{\gamma^{2}} b_{k} s_{k}\left(1-s_{k} \frac{\lambda_{k}}{\lambda_{4}}\right)\left(e^{\frac{\gamma x_{s}^{*}}{x_{k} \lambda_{k}}}-1\right)\right) \widetilde{D}(\gamma)
$$

is positive definite, with $\left(b_{1}, b_{2}, b_{3}\right)^{\top}, D, \widetilde{D}$ and $K$ defined in $(11)$, then the steady state $\left(\left(H^{*}, Q^{*}\right)^{\top}, x_{s}^{*}\right)$ is locally exponentially stable for the $H^{2}$-norm with decay rate $\gamma / 4$.

Remark 2. Note that it is not obvious that there always exists $G$ such that $K$ and $\left(b_{1}, b_{2}, b_{3}\right)^{\top}$ defined in (11) satisfy (18)-(19). We will prove in details that such $G$ indeed exists in Appendix A

\section{Well-posedness of the system}

In this section, we prove the well-posedness of the Saint-Venant equations (1) with the hydraulic jump conditions (3) and (4), the boundary feedback control conditions (9) and initial condition (12). We have the following well-posedness theorem.

Theorem 2.1. For any $T>0$, there exists $\delta(T)>0$ such that, for any given initial condition 12 . satisfying the first order compatibility conditions and

$$
\begin{aligned}
& \left|\left(H_{0}-H_{1}^{*}, Q_{0}-Q^{*}\right)^{\top}\right|_{H^{2}\left(\left(0, x_{s, 0}\right) ; \mathbb{R}^{2}\right)}+\left|\left(H_{0}-H_{2}^{*}, Q_{0}-Q^{*}\right)^{\top}\right|_{H^{2}\left(\left(x_{s, 0}, L\right) ; \mathbb{R}^{2}\right)} \leq \delta(T), \\
& \left|x_{s, 0}-x_{s}^{*}\right| \leq \delta(T),
\end{aligned}
$$


the system (1), (3), (4), (9) and (12) has a unique solution $(H, Q)^{\top} \in C^{0}\left([0, T] ; H^{2}\left(\left(0, x_{s, 0}\right) ; \mathbb{R}^{2}\right) \cap\right.$ $\left.H^{2}\left(\left(x_{s, 0}, L\right) ; \mathbb{R}^{2}\right)\right)$ and $x_{s} \in C^{1}([0, T])$. Moreover, the following estimate holds for any $t \in[0, T]$

$$
\begin{gathered}
\left|\left(H(t, \cdot)-H_{1}^{*}, Q(t, \cdot)-Q^{*}\right)^{\top}\right|_{H^{2}\left(\left(0, x_{s}(t)\right) ; \mathbb{R}^{2}\right)} \\
+\left|\left(H(t, \cdot)-H_{2}^{*}, Q(t, \cdot)-Q^{*}\right)^{\top}\right|_{H^{2}\left(\left(x_{s}(t), L\right) ; \mathbb{R}^{2}\right)}+\left|x_{s}(t)-x_{s}^{*}\right| \\
\leq C(T)\left(\left|\left(H_{0}-H_{1}^{*}, Q_{0}-Q^{*}\right)^{\top}\right|_{H^{2}\left(\left(0, x_{s, 0}\right) ; \mathbb{R}^{2}\right)}\right. \\
\left.\quad+\left|\left(H_{0}-H_{2}^{*}, Q_{0}-Q^{*}\right)^{\top}\right|_{H^{2}\left(\left(x_{s, 0}, L\right) ; \mathbb{R}^{2}\right)}+\left|x_{s, 0}-x_{s}^{*}\right|\right) .
\end{gathered}
$$

Proof. One can see that the shock location $x_{s}$ depends on $t$ in general. In order to avoid the timevarying domains $\left[0, x_{s}(t)\right]$ and $\left[x_{s}(t), L\right]$, under the assumption that $x_{s} \in C^{0}([0, T])$, we perform, as in [12, 20, a transformation of the space coordinate $x$ which allows to define new state variables on the fixed domain $\left[0, x_{s}^{*}\right]$ as follows:

$$
\begin{aligned}
& H_{1}(t, x)=H\left(t, x \frac{x_{s}}{x_{s}^{*}}\right), \\
& Q_{1}(t, x)=Q\left(t, x \frac{x_{s}}{x_{s}^{*}}\right), \\
& H_{2}(t, x)=H\left(t, L+x \frac{x_{s}-L}{x_{s}^{*}}\right), \\
& Q_{2}(t, x)=Q\left(t, L+x \frac{x_{s}-L}{x_{s}^{*}}\right) .
\end{aligned}
$$

Let us denote by $h_{i}$ and $q_{i}$ the deviations

$$
h_{i}=H_{i}-H_{i}^{*}, \quad q_{i}=Q_{i}-Q^{*}, \quad i=1,2 .
$$

Then, the system (11), (3) and (4) is equivalent to the following $4 \times 4$ system, which is diagonalisable by blocks and defined on $\mathbb{R}^{+} \times\left[0, x_{s}^{*}\right]$ :

$$
\begin{aligned}
& \partial_{t} h_{1}-\left(x \frac{\dot{x}_{s}}{x_{s}^{*}}\right) \frac{x_{s}^{*}}{x_{s}} \partial_{x} h_{1}+\frac{x_{s}^{*}}{x_{s}} \partial_{x} q_{1}=0 \\
& \partial_{t} q_{1}+\left(\frac{2\left(q_{1}+Q^{*}\right)}{h_{1}+H_{1}^{*}}-x \frac{\dot{x}_{s}}{x_{s}^{*}}\right) \frac{x_{s}^{*}}{x_{s}} \partial_{x} q_{1}+\left(g\left(h_{1}+H_{1}^{*}\right)-\frac{\left(q_{1}+Q^{*}\right)^{2}}{\left(h_{1}+H_{1}^{*}\right)^{2}}\right) \frac{x_{s}^{*}}{x_{s}} \partial_{x} h_{1}=0, \\
& \partial_{t} h_{2}+\left(x \frac{\dot{x}_{s}}{x_{s}^{*}}\right) \frac{x_{s}^{*}}{L-x_{s}} \partial_{x} h_{2}-\frac{x_{s}^{*}}{L-x_{s}} \partial_{x} q_{2}=0, \\
& \partial_{t} q_{2}-\left(\frac{2\left(q_{2}+Q^{*}\right)}{h_{2}+H_{2}^{*}}-x \frac{\dot{x}_{s}}{x_{s}^{*}}\right) \frac{x_{s}^{*}}{L-x_{s}} \partial_{x} q_{2}-\left(g\left(h_{2}+H_{2}^{*}\right)-\frac{\left(q_{2}+Q^{*}\right)^{2}}{\left(h_{2}+H_{2}^{*}\right)^{2}}\right) \frac{x_{s}^{*}}{L-x_{s}} \partial_{x} h_{2}=0,
\end{aligned}
$$

where

$$
\dot{x}_{s}=\frac{q_{2}\left(t, x_{s}^{*}\right)-q_{1}\left(t, x_{s}^{*}\right)}{h_{2}\left(t, x_{s}^{*}\right)-h_{1}\left(t, x_{s}^{*}\right)+H_{2}^{*}-H_{1}^{*}}
$$

and with, from the jump condition (4), the following boundary condition at $x=x_{s}^{*}$ :

$$
\left(q_{2}-q_{1}\right)^{2}=\left(h_{2}-h_{1}+H_{2}^{*}-H_{1}^{*}\right)\left(\frac{\left(q_{2}+Q^{*}\right)^{2}}{h_{2}+H_{2}^{*}}+\frac{g}{2}\left(h_{2}+H_{2}^{*}\right)^{2}-\frac{\left(q_{1}+Q^{*}\right)^{2}}{h_{1}+H_{1}^{*}}-\frac{g}{2}\left(h_{1}+H_{1}^{*}\right)^{2}\right) .
$$

Now, we introduce the following Riemann coordinates

$$
\mathbf{u}=\left(\begin{array}{l}
u_{1} \\
u_{2} \\
u_{3} \\
u_{4}
\end{array}\right)=\left(\begin{array}{cc}
S_{1} & 0 \\
0 & S_{2}
\end{array}\right)\left(\begin{array}{l}
h_{1} \\
q_{1} \\
h_{2} \\
q_{2}
\end{array}\right)
$$

with

$$
S_{1}=\left(\begin{array}{cc}
\frac{1}{\lambda_{1}} & \frac{1}{\lambda_{2}} \\
1 & 1
\end{array}\right)^{-1}, \quad S_{2}=\left(\begin{array}{cc}
-\frac{1}{\lambda_{3}} & \frac{1}{\lambda_{4}} \\
1 & 1
\end{array}\right)^{-1}
$$


and $\lambda_{i}$ defined in $(6),(7)$. Then the system $(25)$ can be rewritten as

$$
\mathbf{u}_{t}+\left(\Lambda\left(x_{s}\right)+A\left(\mathbf{u}, x_{s}\right)+x \dot{x}_{s} B\left(x_{s}\right)\right) \mathbf{u}_{x}=0
$$

where

$$
\Lambda=\left(\begin{array}{cccc}
\frac{x_{s}^{*}}{x_{s}} \lambda_{1} & 0 & 0 & 0 \\
0 & \frac{x_{s}^{*}}{x_{s}} \lambda_{2} & 0 & 0 \\
0 & 0 & \frac{x_{s}^{*}}{L-x_{s}} \lambda_{3} & 0 \\
0 & 0 & 0 & -\frac{x_{s}^{*}}{L-x_{s}} \lambda_{4}
\end{array}\right)
$$

and where $A, B$ are two matrices of class $C^{2}$ that can be obtained by direct computations (omitted here for simplicity) and such that $A$ satisfies $A\left(\mathbf{0}, x_{s}\right)=0$. Using the change of coordinates (28), equation (26) becomes:

$$
\dot{x}_{s}=\frac{u_{1}\left(t, x_{s}^{*}\right)+u_{2}\left(t, x_{s}^{*}\right)-u_{3}\left(t, x_{s}^{*}\right)-u_{4}\left(t, x_{s}^{*}\right)}{\sum_{i=1}^{3} \frac{u_{i}\left(t, x_{s}^{*}\right)}{\lambda_{i}}-\frac{u_{4}\left(t, x_{s}^{*}\right)}{\lambda_{4}}+\left(H_{1}^{*}-H_{2}^{*}\right)}
$$

and the boundary condition (27) becomes:

$$
\frac{2 Q^{*}}{H_{2}^{*}}\left(u_{3}+u_{4}\right)-\frac{2 Q^{*}}{H_{1}^{*}}\left(u_{1}+u_{2}\right)+\left(g H_{2}^{*}-\frac{Q^{* 2}}{H_{2}^{* 2}}\right)\left(\frac{u_{4}}{\lambda_{4}}-\frac{u_{3}}{\lambda_{3}}\right)-\left(g H_{1}^{*}-\frac{Q^{* 2}}{H_{1}^{* 2}}\right)\left(\frac{u_{1}}{\lambda_{1}}+\frac{u_{2}}{\lambda_{2}}\right)=O\left(\left|\mathbf{u}\left(t, x_{s}^{*}\right)\right|^{2}\right) .
$$

Here and hereafter, $O(s)$ (with $s \geq 0$ ) means that for any $\varepsilon>0$, there exists $C_{1}>0$ such that

$$
(s \leq \varepsilon) \Longrightarrow\left(|O(s)| \leq C_{1} s\right) .
$$

With the expression of the eigenvalues given by (6) and (7), (33) becomes

$$
\lambda_{4} u_{4}\left(t, x_{s}^{*}\right)=\lambda_{1} u_{1}\left(t, x_{s}^{*}\right)+\lambda_{2} u_{2}\left(t, x_{s}^{*}\right)+\lambda_{3} u_{3}\left(t, x_{s}^{*}\right)+O\left(\left|\mathbf{u}\left(t, x_{s}^{*}\right)\right|^{2}\right) .
$$

Using (23), 24, (28) and (34), the boundary conditions $(9)$ now become

$$
\left(\begin{array}{l}
u_{1}(t, 0) \\
u_{2}(t, 0) \\
u_{3}(t, 0)
\end{array}\right)=\mathcal{B}\left(\left(\begin{array}{l}
u_{1}\left(t, x_{s}^{*}\right) \\
u_{2}\left(t, x_{s}^{*}\right) \\
u_{3}\left(t, x_{s}^{*}\right)
\end{array}\right), u_{4}(t, 0), x_{s}-x_{s}^{*}\right),
$$

where $\mathcal{B}=\left(B_{1}, B_{2}, B_{3}\right)^{\top}: \mathbb{R}^{3} \times \mathbb{R} \times \mathbb{R} \rightarrow \mathbb{R}^{3}$ is of class $C^{2}$ and where $B_{1}$ and $B_{2}$ are defined by

$$
\begin{aligned}
& B_{1}=\left(\lambda_{2} G_{1}\left(\mathbf{u}\left(t, x_{s}^{*}\right), x_{s}\right)-G_{2}\left(\mathbf{u}\left(t, x_{s}^{*}\right), x_{s}\right)\right) \frac{\lambda_{1}}{\lambda_{2}-\lambda_{1}}, \\
& B_{2}=\left(\lambda_{1} G_{1}\left(\mathbf{u}\left(t, x_{s}^{*}\right), x_{s}\right)-G_{2}\left(\mathbf{u}\left(t, x_{s}^{*}\right), x_{s}\right)\right) \frac{\lambda_{2}}{\lambda_{1}-\lambda_{2}} .
\end{aligned}
$$

To define $B_{3}$, from the boundary conditions $(9)$ and the change of variables $(24)$, (28), we have

$$
\begin{aligned}
u_{3}(t, 0)=\frac{-\lambda_{4} \lambda_{3}}{\lambda_{3}+\lambda_{4}}\left(\frac{u_{4}(t, 0)}{\lambda_{4}}-\frac{u_{3}(t, 0)}{\lambda_{3}}\right)+\frac{\lambda_{3}}{\lambda_{3}+\lambda_{4}} G_{3}\left(\mathbf{u}\left(t, x_{s}^{*}\right), x_{s}\right) & -\frac{\lambda_{3}}{\lambda_{3}+\lambda_{4}} G_{4}\left(\frac{u_{4}(t, 0)}{\lambda_{4}}-\frac{u_{3}(t, 0)}{\lambda_{3}}\right) .
\end{aligned}
$$

From condition 10 , applying the implicit function theorem, one obtains

$$
B_{3}=\mathcal{F}\left(u_{4}(t, 0), G_{3}\left(\mathbf{u}\left(t, x_{s}^{*}\right), x_{s}\right)\right)
$$

in a neighborhood of $\mathbf{u}=0$ with

$$
\mathcal{F}(0,0)=0, \quad \partial_{1} \mathcal{F}(0,0)=0, \quad \partial_{2} \mathcal{F}(0,0)=\frac{\lambda_{3}}{\lambda_{3}+\lambda_{4}},
$$

where $\partial_{i} \mathcal{F}, i=1,2$, denote the partial derivative of $\mathcal{F}$ with respect to its $i$-th variable. 
Remark 3. For simplicity, in (36)-(39), we have used the following slight abuse of notation adapted from (9):

$$
G_{i}\left(\mathbf{u}\left(t, x_{s}^{*}\right), x_{s}\right)=G_{i}\left(\begin{array}{c}
u_{1}\left(t, x_{s}^{*}\right)+u_{2}\left(t, x_{s}^{*}\right) \\
u_{3}\left(t, x_{s}^{*}\right)+u_{4}\left(t, x_{s}^{*}\right) \\
\frac{u_{1}\left(t, x_{s}^{*}\right)}{\lambda_{1}}+\frac{u_{2}\left(t, x_{s}^{*}\right)}{\lambda_{2}} \\
x_{s}-x_{s}^{*}
\end{array}\right), \quad i=1,2,3 .
$$

From (23), (24), (28) and (34), one can see that, as expressed in (35), $\mathcal{B}$ only depends on $u_{i}\left(t, x_{s}^{*}\right)$, $i=1,2,3, u_{4}(t, 0)$ and $x_{s}-x_{s}^{*}$ because from (34) $u_{4}\left(t, x_{s}^{*}\right)$ can be considered as a function of $u_{i}\left(t, x_{s}^{*}\right)$, $i=1,2,3$.

The initial condition 12 becomes

$$
\begin{aligned}
& \mathbf{u}(0, x)=\mathbf{u}_{0}(x)=\left(u_{10}(x), u_{20}(x), u_{30}(x), u_{40}(x)\right)^{\top}, \\
& x_{s}(0)=x_{s, 0}
\end{aligned}
$$

that satisfies the first order compatibility conditions corresponding to 35 . Thus, to study the wellposedness of (1), (3), (4), (9) and $(12)$ is equivalent to study the well-posedness of $(30),(32),(34)$, (35) and (42). We have the following lemma from which one can easily obtain Theorem 2.1.

Lemma 2.1. For any $T>0$, there exists $\delta(T)>0$ such that, for any $x_{s, 0} \in(0, L)$ and $\mathbf{u}_{0} \in$ $H^{2}\left(\left(0, x_{s}^{*}\right) ; \mathbb{R}^{4}\right)$ satisfying the first order compatibility conditions and

$$
\left|\mathbf{u}_{0}\right|_{H^{2}\left(\left(0, x_{s}^{*}\right) ; \mathbb{R}^{4}\right)} \leq \delta(T) \text { and }\left|x_{s, 0}-x_{s}^{*}\right| \leq \delta(T),
$$

the system (30), (32), (34), (35) and 42 has a unique solution $\mathbf{u} \in C^{0}\left([0, T] ; H^{2}\left(\left(0, x_{s}^{*}\right) ; \mathbb{R}^{4}\right)\right)$ and $x_{s} \in C^{1}([0, T])$. Moreover, the following estimate holds for any $t \in[0, T]$

$$
|\mathbf{u}(t, \cdot)|_{H^{2}\left(\left(0, x_{s}^{*}\right) ; \mathbb{R}^{4}\right)}+\left|x_{s}(t)-x_{s}^{*}\right| \leq C(T)\left(\left|\mathbf{u}_{0}\right|_{H^{2}\left(\left(0, x_{s}^{*}\right) ; \mathbb{R}^{4}\right)}+\left|x_{s, 0}-x_{s}^{*}\right|\right) .
$$

Remark 4. For the proof of Lemma 2.1, we refer to [5, Appendix], where the well-posedness of a $2 \times 2$ nonlinear hyperbolic system coupled with an ODE was studied. But the proof there can be easily adapted to the $4 \times 4$ nonlinear hyperbolic system coupled with an ODE. Noticing that $A\left(\mathbf{0}, x_{s}\right)=0$ and that, from $(32), \dot{x}_{s}=0$ when $\mathbf{u}=\mathbf{0}$, one has

$$
\Lambda\left(x_{s}\right)+A\left(\mathbf{u}, x_{s}\right)+x \dot{x}_{s} B\left(x_{s}\right)=\Lambda\left(x_{s}\right)
$$

when $\mathbf{u}=\mathbf{0}$. Thus, 30 is indeed strictly hyperbolic provided that $|\mathbf{u}|_{C^{0}\left([0, T] ; H^{2}\left(\left(0, x_{s}^{*}\right) ; \mathbb{R}^{4}\right)\right)}$ is small enough and can be diagonalized in a neighbourhood of $\mathbf{u}=\mathbf{0}$. Then we can perform similar fixed point argument as in [5, Appendix] by carefully estimating the related norms of the solution along the characteristic curves. The $C^{1}$ regularity of $x_{s}$ is then obtained directly from $(32)$. We omit the details.

This completes the proof of Theorem 2.1.

\section{Exponential stability of the steady state for the $H^{2}$-norm}

In this section we prove Theorem 1.1 .

Proof of Theorem 1.1. It is worth noticing that due to the equivalence of the system (1), (3), (4), (9) and the system (30), (32), (34) and (35), one only needs to prove the exponential stability of the null-steady state of the system (30), (32), (34) and (35) for the $H^{2}$-norm.

Motivated by [8, see also [2, Section 4.4], and by [5], we introduce the following Lyapunov function:

$$
V\left(\mathbf{u}, x_{s}\right)=V_{1}(\mathbf{u})+V_{2}(\mathbf{u})+V_{3}(\mathbf{u})+V_{4}\left(\mathbf{u}, x_{s}\right)+V_{5}\left(\mathbf{u}, x_{s}\right)+V_{6}\left(\mathbf{u}, x_{s}\right),
$$


where:

$$
\begin{gathered}
V_{1}(\mathbf{u})=\int_{0}^{x_{s}^{*}} \sum_{i=1}^{4} p_{i} e^{-\frac{\mu}{x_{i} \lambda_{i}} x} u_{i}^{2} d x, \\
V_{2}(\mathbf{u})=\int_{0}^{x_{s}^{*}} \sum_{i=1}^{4} p_{i} e^{-\frac{\mu}{x_{i} \lambda_{i}} x} u_{i t}^{2} d x, \\
V_{3}(\mathbf{u})=\int_{0}^{x_{s}^{*}} \sum_{i=1}^{4} p_{i} e^{-\frac{\mu}{x_{i} \lambda_{i}} x} u_{i t t}^{2} d x, \\
V_{4}\left(\mathbf{u}, x_{s}\right)=\int_{0}^{x_{s}^{*}} \sum_{i=1}^{3} \frac{p_{i}^{\prime}}{\lambda_{i}} e^{-\frac{\mu}{x_{i} \lambda_{i}} x} u_{i}(t, x)\left(x_{s}-x_{s}^{*}\right) d x+C_{0}\left(x_{s}-x_{s}^{*}\right)^{2}, \\
V_{5}\left(\mathbf{u}, x_{s}\right)=\int_{0}^{x_{s}^{*}} \sum_{i=1}^{3} \frac{p_{i}^{\prime}}{\lambda_{i}} e^{-\frac{\mu}{x_{i} \lambda_{i}} x} u_{i t}(t, x) \dot{x}_{s} d x+C_{0}\left(\dot{x}_{s}\right)^{2}, \\
V_{6}\left(\mathbf{u}, x_{s}\right)=\int_{0}^{x_{s}^{*}} \sum_{i=1}^{3} \frac{p_{i}^{\prime}}{\lambda_{i}} e^{-\frac{\mu}{x_{i} \lambda_{i}} x} u_{i t t}(t, x) \ddot{x}_{s} d x+C_{0}\left(\ddot{x}_{s}\right)^{2},
\end{gathered}
$$

where $p_{i}$ and $C_{0}$ are positive constants that shall be determined later on, while $p_{i}^{\prime}$ are constants, not necessarily positive, which will also be determined later on. Besides we impose $C_{0}>3 / 2$ and we recall that $x_{1}=x_{2}=1, x_{3}=x_{s}^{*} /\left(L-x_{s}^{*}\right)$ and $x_{4}=x_{s}^{*} /\left(x_{s}^{*}-L\right)$. In the following we may denote for simplicity $V_{i}:=V_{i}\left(\mathbf{u}, x_{s}\right)$ and $|\mathbf{u}|_{H^{2}}:=|\mathbf{u}(t, \cdot)|_{H^{2}\left(\left(0, x_{s}^{*}\right) ; \mathbb{R}^{4}\right)}$ in the computations. Similarly to what is done in [5], from the Cauchy-Schwarz inequality and as $C_{0}>3 / 2$, it can be shown that the Lyapunov function $V$ considered here is equivalent to $\left(|\mathbf{u}|_{H^{2}}+\left|x_{s}-x_{s}^{*}\right|\right)^{2}$ provided that $|\mathbf{u}|_{H^{2}}+\left|x_{s}-x_{s}^{*}\right|$ is small enough and that

$$
\max _{i}\left(\frac{p_{i}^{\prime 2} x_{i}}{\mu \lambda_{i} p_{i}}\left(1-e^{-\frac{\mu}{x_{i} \lambda_{i}} x_{s}^{*}}\right)\right)<2 .
$$

This means that, under condition (52), there exists $\bar{\rho}>0$ and $\bar{C}$ such that, for every $T>0$ and $\mathbf{u} \in C^{0}\left([0, T] ; H^{2}\left(\left(0, x_{s}^{*}\right) ; \mathbb{R}^{4}\right)\right)$ and for every $x_{s} \in C^{1}([0, T])$ solution of the system (30), (32), (34) and (35), if $|\mathbf{u}|_{H^{2}}+\left|x_{s}-x_{s}^{*}\right| \leq \bar{\rho}$

$$
\frac{1}{\bar{C}}\left(|\mathbf{u}|_{H^{2}}+\left|x_{s}-x_{s}^{*}\right|\right)^{2} \leq V\left(\mathbf{u}, x_{s}\right) \leq \bar{C}\left(|\mathbf{u}|_{H^{2}}+\left|x_{s}-x_{s}^{*}\right|\right)^{2} .
$$

This can be proved by direct estimations (see [5] for more details).

From the boundary condition $(35)$, as $\mathcal{B}$ is of class $C^{2}$, we have

$$
\mathbf{v}(t, 0)=\partial_{1} \mathcal{B}(\mathbf{0}, 0,0) \mathbf{v}\left(t, x_{s}^{*}\right)+\partial_{2} \mathcal{B}(\mathbf{0}, 0,0) u_{4}(t, 0)+\partial_{3} \mathcal{B}(\mathbf{0}, 0,0)\left(x_{s}-x_{s}^{*}\right)+O\left(\left(|\mathbf{u}|_{H^{2}}+\left|x_{s}-x_{s}^{*}\right|\right)^{2}\right),
$$

where $\mathbf{v}=\left(u_{1}, u_{2}, u_{3}\right)^{\top}$ is the vector of the components of $\mathbf{u}$ on which the feedback (35) applies. This notation is practical as it isolates $u_{1}, u_{2}$ and $u_{3}$ from $u_{4}$ on which we have no control and whose boundary condition is imposed by the condition (34). In (54), the notation $\partial_{1} \mathcal{B}$ is the $3 \times 3$ Jacobian matrix of the vector-valued function $\mathcal{B}$ with respect to its first variable which is a 3 -D vector (see the expression of $\mathcal{B}$ in (35)). From $(36)-(40)$, one can check that $\partial_{2} \mathcal{B}(\mathbf{0}, 0,0) \equiv \mathbf{0}$. Moreover, from (36) - 395), noticing (40), it can be verified that the matrix $K$ and the vector $\left(b_{1}, b_{2}, b_{3}\right)^{\top}$ defined in (11) satisfy

$$
K=\left(k_{i j}\right)_{(i, j) \in\{1,2,3\}^{2}}=\partial_{1} \mathcal{B}(\mathbf{0}, 0,0), \quad \partial_{3} \mathcal{B}(\mathbf{0}, 0,0)=\left(b_{1}, b_{2}, b_{3}\right)^{\top} .
$$

Let $\bar{T}>0$ be given and let $x_{s, 0} \in(0, L)$ and $\mathbf{u}_{0} \in H^{2}\left(\left(0, x_{s}^{*}\right) ; \mathbb{R}^{4}\right)$ satisfying the first order compatibility conditions and (43). Let $\mathbf{u} \in C^{0}\left([0, \bar{T}] ; H^{2}\left(\left(0, x_{s}^{*}\right) ; \mathbb{R}^{4}\right)\right)$ and $x_{s} \in C^{1}([0, \bar{T}])$ be the solution of the system (30), (32), (34)-(42). Let us start with the case where $\mathbf{u}$ is of class $C^{3}$. Taking the time derivative of $V_{1}$ along this solution and integrating by parts, we obtain

$$
\frac{d V_{1}}{d t}=-\mu V_{1}-\left[\sum_{i=1}^{4} p_{i} x_{i} \lambda_{i} e^{-\frac{\mu}{x_{i} \lambda_{i}} x} u_{i}^{2}\right]_{0}^{x_{s}^{*}}+O\left(\left(|\mathbf{u}|_{H^{2}}+\left|x_{s}-x_{s}^{*}\right|\right)^{3}\right) .
$$


By differentiating $(30)$, similarly as $(56)$, we can obtain

$$
\frac{d V_{2}}{d t}=-\mu V_{2}-\left[\sum_{i=1}^{4} p_{i} x_{i} \lambda_{i} e^{-\frac{\mu}{x_{i} \lambda_{i}} x} u_{i t}^{2}\right]_{0}^{x_{s}^{*}}+O\left(\left(|\mathbf{u}|_{H^{2}}+\left|x_{s}-x_{s}^{*}\right|\right)^{3}\right) .
$$

Now, let us deal with the $V_{3}$ term. To that end, we derive from 30 that

$$
\begin{aligned}
& \mathbf{u}_{t t t}+\Lambda\left(x_{s}\right) \mathbf{u}_{t t x}+2 \dot{x}_{s} \Lambda^{\prime}\left(x_{s}\right) \mathbf{u}_{t x}+\left(\Lambda^{\prime \prime}\left(x_{s}\right)\left(\dot{x}_{s}\right)^{2}+\Lambda^{\prime}\left(x_{s}\right) \ddot{x}_{s}\right) \mathbf{u}_{x} \\
& +\left(A\left(\mathbf{u}, x_{s}\right) \mathbf{u}_{x}\right)_{t t}+x \dddot{x}_{s} B\left(x_{s}\right) \mathbf{u}_{x}+2 x \ddot{x}_{s}\left(B\left(x_{s}\right) \mathbf{u}_{x}\right)_{t}+x \dot{x}_{s}\left(B\left(x_{s}\right) \mathbf{u}_{x}\right)_{t t}=0 .
\end{aligned}
$$

Thus,

$$
\begin{aligned}
\frac{d V_{3}}{d t}= & -\mu V_{3}-\left[\sum_{i=1}^{4} p_{i} x_{i} \lambda_{i} e^{-\frac{\mu}{x_{i} \lambda_{i}} x} u_{i t t}^{2}\right]_{0}^{x_{s}^{*}}-\int_{0}^{x_{s}^{*}} \sum_{i=1}^{4} 2 p_{i} e^{-\frac{\mu}{x_{i} \lambda_{i}} x} x_{x_{s}} u_{i t t}\left(\sum_{j=1}^{4} B_{i j} u_{j x}\right) d x \\
& +O\left(\left(|\mathbf{u}|_{H^{2}}+\left|x_{s}-x_{s}^{*}\right|\right)^{3}\right) .
\end{aligned}
$$

We observe that now $\dddot{x}_{s}$ appears. As $\dddot{x}_{s}$ is proportional to $\mathbf{u}_{t t}\left(x_{s}^{*}\right)$, it can not be bounded by $|\mathbf{u}|_{H^{2}}$. However, we can use Young's inequality to compensate it with the boundary terms. Using (54), one has

$$
\begin{aligned}
\frac{d V_{3}}{d t} \leq & -\mu V_{3}-\sum_{i=1}^{4}\left(\left(p_{i} x_{i} \lambda_{i} e^{-\frac{\mu x_{s}^{*}}{x_{i} \lambda_{i}}}+O\left(|\mathbf{u}|_{H^{2}}\right)\right) u_{i t t}^{2}\left(x_{s}^{*}\right)-u_{i t t}^{2}(0)\right) \\
& +O\left(\left(|\mathbf{u}|_{H^{2}}+\left|x_{s}-x_{s}^{*}\right|\right)^{3}\right) .
\end{aligned}
$$

Differentiating (49), from (30), one has

$$
\begin{aligned}
\frac{d V_{4}}{d t}= & \left(x_{s}-x_{s}^{*}\right) \int_{0}^{x_{s}^{*}} \sum_{i=1}^{3} \frac{p_{i}^{\prime}}{\lambda_{i}} e^{-\frac{\mu}{x_{i} \lambda_{i}} x} u_{i t}(t, x) d x \\
& +\dot{x}_{s} \int_{0}^{x_{s}^{*}} \sum_{i=1}^{3} \frac{p_{i}^{\prime}}{\lambda_{i}} e^{-\frac{\mu}{x_{i} \lambda_{i}} x} u_{i}(t, x) d x+2 C_{0} \dot{x}_{s}\left(x_{s}-x_{s}^{*}\right) \\
= & -\left(x_{s}-x_{s}^{*}\right) \int_{0}^{x_{s}^{*}} \sum_{i=1}^{3} x_{i} p_{i}^{\prime} e^{-\frac{\mu}{x_{i} \lambda_{i}} x} u_{i x}(t, x) d x \\
& +d\left(u_{1}\left(x_{s}^{*}\right)+u_{2}\left(x_{s}^{*}\right)-u_{3}\left(x_{s}^{*}\right)-u_{4}\left(x_{s}^{*}\right)\right) \int_{0}^{x_{s}^{*}} \sum_{i=1}^{3} \frac{p_{i}^{\prime}}{\lambda_{i}} e^{-\frac{\mu}{x_{i} \lambda_{i}} x} u_{i}(t, x) d x \\
& +2 d C_{0}\left(x_{s}-x_{s}^{*}\right)\left(u_{1}\left(x_{s}^{*}\right)+u_{2}\left(x_{s}^{*}\right)-u_{3}\left(x_{s}^{*}\right)-u_{4}\left(x_{s}^{*}\right)\right) \\
& +O\left(\left(|\mathbf{u}|_{H^{2}}+\left|x_{s}-x_{s}^{*}\right|\right)^{3}\right),
\end{aligned}
$$

where we recall that $d=\left(H_{1}^{*}-H_{2}^{*}\right)^{-1}<0$ is defined in 11 . Thus, integrating by parts and using (34),

$$
\begin{aligned}
\frac{d V_{4}}{d t}=-\left(x_{s}-x_{s}^{*}\right) & {\left[\sum_{i=1}^{3} x_{i} p_{i}^{\prime} e^{-\frac{\mu}{x_{i} \lambda_{i}} x} u_{i}(t, x)\right]_{0}^{x_{s}^{*}}-\mu\left(V_{4}-C_{0}\left(x_{s}-x_{s}^{*}\right)^{2}\right) } \\
+ & d\left(u_{1}\left(x_{s}^{*}\right)\left(1-\frac{\lambda_{1}}{\lambda_{4}}\right)+u_{2}\left(x_{s}^{*}\right)\left(1-\frac{\lambda_{2}}{\lambda_{4}}\right)-u_{3}\left(x_{s}^{*}\right)\left(1+\frac{\lambda_{3}}{\lambda_{4}}\right)\right) \\
& \left(2 C_{0}\left(x_{s}-x_{s}^{*}\right)+\int_{0}^{x_{s}^{*}} \sum_{i=1}^{3} \frac{p_{i}^{\prime}}{\lambda_{i}} e^{-\frac{\mu}{x_{i} \lambda_{i}} x} u_{i}(t, x) d x\right)+O\left(\left(|\mathbf{u}|_{H^{2}}+\left|x_{s}-x_{s}^{*}\right|\right)^{3}\right)
\end{aligned}
$$


Similarly for $V_{5}$, from 30 , one has

$$
\begin{aligned}
\frac{d V_{5}}{d t}= & -\dot{x}_{s}\left[\sum_{i=1}^{3} x_{i} p_{i}^{\prime} e^{-\frac{\mu}{x_{i} \lambda_{i}} x} u_{i t}(t, x)\right]_{0}^{x_{s}^{*}}-\mu\left(V_{5}-C_{0}\left(\dot{x}_{s}\right)^{2}\right) \\
& +d\left(\sum_{i=1}^{3}\left(1-s_{i} \frac{\lambda_{i}}{\lambda_{4}}\right) s_{i} u_{i t}\left(x_{s}^{*}\right)\right)\left(2 C_{0} \dot{x}_{s}+\int_{0}^{x_{s}^{*}} \sum_{i=1}^{3} \frac{p_{i}^{\prime}}{\lambda_{i}} e^{-\frac{\mu}{x_{i} \lambda_{i}} x} u_{i t}(t, x) d x\right) \\
& +O\left(\left(|\mathbf{u}|_{H^{2}}+\left|x_{s}-x_{s}^{*}\right|\right)^{3}\right) .
\end{aligned}
$$

By 58 , for $V_{6}$, one has

$$
\begin{aligned}
\frac{d V_{6}}{d t}= & -\ddot{x}_{s}\left[\sum_{i=1}^{3} x_{i} p_{i}^{\prime} e^{-\frac{\mu}{x_{i} \lambda_{i}} x} u_{i t t}(t, x)\right]_{0}^{x_{s}^{*}}-\mu\left(V_{6}-C_{0}\left(\ddot{x}_{s}\right)^{2}\right) \\
& +d\left(\sum_{i=1}^{3}\left(1-s_{i} \frac{\lambda_{i}}{\lambda_{4}}\right) s_{i} u_{i t t}\left(x_{s}^{*}\right)\right)\left(2 C_{0} \ddot{x}_{s}+\int_{0}^{x_{s}^{*}} \sum_{i=1}^{3} \frac{p_{i}^{\prime}}{\lambda_{i}} e^{-\frac{\mu}{x_{i} \lambda_{i}} x} u_{i t t}(t, x) d x\right) \\
& -\int_{0}^{x_{s}^{*}} \sum_{i=1}^{3} \frac{p_{i}^{\prime}}{\lambda_{i}} e^{-\frac{\mu}{x_{i} \lambda_{i}} x} x \dddot{x}_{s}\left(\sum_{j=1}^{4} B_{i j} u_{j x}\right)\left(x_{s}-x_{s}^{*}\right) d x+O\left(\left(|\mathbf{u}|_{H^{2}}+\left|x_{s}-x_{s}^{*}\right|\right)^{3}\right) .
\end{aligned}
$$

Dealing with the $\dddot{x}_{s}$ term in (64) similarly as for $V_{3}$, we have

$$
\begin{aligned}
\frac{d V_{6}}{d t}= & -\ddot{x}_{s} \sum_{i=1}^{3}\left(\left(x_{i} p_{i}^{\prime} e^{-\frac{\mu}{x_{i} \lambda_{i}} x_{s}^{*}}+O\left(|\mathbf{u}|_{H^{2}}\right)\right) u_{i t t}\left(x_{s}^{*}\right)-x_{i} p_{i}^{\prime} u_{i t t}(0)\right)-\mu\left(V_{6}-C_{0}\left(\ddot{x}_{s}\right)^{2}\right) \\
& +d\left(\sum_{i=1}^{3}\left(1-s_{i} \frac{\lambda_{i}}{\lambda_{4}}\right) s_{i} u_{i t t}\left(x_{s}^{*}\right)\right)\left(2 C_{0} \ddot{x}_{s}+\int_{0}^{x_{s}^{*}} \sum_{i=1}^{3} \frac{p_{i}^{\prime}}{\lambda_{i}} e^{-\frac{\mu}{x_{i} \lambda_{i}} x} u_{i t t}(t, x) d x\right) \\
& +O\left(\left(|\mathbf{u}|_{H^{2}}+\left|x_{s}-x_{s}^{*}\right|\right)^{3}\right) .
\end{aligned}
$$

Note that $V_{2}+V_{5}$ has the same structure as $V_{1}+V_{4}$ with $u_{i}$ and $x_{s}-x_{s}^{*}$ being replaced by $u_{i t}$ and $\dot{x}_{s}$ respectively. The same applies for $V_{3}+V_{6}$ by replacing $u_{i}$ and $x_{s}-x_{s}^{*}$ in $V_{1}+V_{4}$ with $u_{i t t}$ and $\ddot{x}_{s}$ respectively. Hence, we only need to analyze $V_{1}+V_{4}$. From (56) and $(62)$, recalling that $s_{i}=1$ if $i \in\{1,2\}$ and $s_{3}=-1$, one has

$$
\begin{aligned}
\frac{d\left(V_{1}+V_{4}\right)}{d t}= & -\left[\sum_{i=1}^{4} p_{i} x_{i} \lambda_{i} e^{-\frac{\mu}{x_{i} \lambda_{i}} x} u_{i}^{2}\right]_{0}^{x_{s}^{*}}-\mu\left(V_{1}+V_{4}\right) \\
& -\left(x_{s}-x_{s}^{*}\right)\left[\sum_{i=1}^{3} x_{i} p_{i}^{\prime} e^{-\frac{\mu}{x_{i} \lambda_{i}} x} u_{i}\right]_{0}^{x_{s}^{*}}+\mu C_{0}\left(x_{s}-x_{s}^{*}\right)^{2} \\
& +d\left(\sum_{i=1}^{3} u_{i}\left(x_{s}^{*}\right) s_{i}\left(1-s_{i} \frac{\lambda_{i}}{\lambda_{4}}\right)\right)\left(2 C_{0}\left(x_{s}-x_{s}^{*}\right)+\int_{0}^{x_{s}^{*}} \sum_{i=1}^{3} \frac{p_{i}^{\prime}}{\lambda_{i}} e^{-\frac{\mu}{x_{i} \lambda_{i}} x_{i}} u_{i}(t, x) d x\right) \\
& +O\left(\left(|\mathbf{u}|_{H^{2}}+\left|x_{s}-x_{s}^{*}\right|\right)^{3}\right) .
\end{aligned}
$$


Using now the boundary conditions (54) and (34), and noticing (55), (66) becomes

$$
\begin{aligned}
\frac{d\left(V_{1}+V_{4}\right)}{d t}= & -\mu\left(V_{1}+V_{4}\right) \\
& -\mathbf{v}\left(x_{s}^{*}\right)^{\top}\left(F\left(x_{s}^{*}, \mu\right)-K^{\top} F(0, \mu) K\right) \mathbf{v}\left(x_{s}^{*}\right)-\frac{x_{4} p_{4}}{\lambda_{4}} e^{-\frac{\mu}{x_{4} \lambda_{4}} x_{s}^{*}}\left(\lambda_{1} u_{1}\left(x_{s}^{*}\right)+\lambda_{2} u_{2}\left(x_{s}^{*}\right)+\lambda_{3} u_{3}\left(x_{s}^{*}\right)\right)^{2} \\
& -\lambda_{4}\left|x_{4}\right| p_{4} u_{4}^{2}(0)+\sum_{i=1}^{3} x_{i} p_{i} \lambda_{i} b_{i}^{2}\left(x_{s}-x_{s}^{*}\right)^{2}+2 \sum_{i=1}^{3} x_{i} p_{i} \lambda_{i} b_{i}\left(\sum_{j=1}^{3} k_{i j} u_{j}\left(x_{s}^{*}\right)\left(x_{s}-x_{s}^{*}\right)\right) \\
& -\left(\sum_{i=1}^{3} u_{i}\left(x_{s}^{*}\right)\left(x_{s}-x_{s}^{*}\right)\left(x_{i} p_{i}^{\prime} e^{-\frac{\mu}{x_{i} \lambda_{i}} x_{s}^{*}}-2 d C_{0} s_{i}\left(1-s_{i} \frac{\lambda_{i}}{\lambda_{4}}\right)\right)-\sum_{j=1}^{3} k_{i j} u_{j}\left(x_{s}^{*}\right)\left(x_{s}-x_{s}^{*}\right) x_{i} p_{i}^{\prime}\right) \\
& +\sum_{i=1}^{3} x_{i} p_{i}^{\prime} b_{i}\left(x_{s}-x_{s}^{*}\right)^{2}+\mu C_{0}\left(x_{s}-x_{s}^{*}\right)^{2} \\
& +d\left(\sum_{i=1}^{3} u_{i}\left(x_{s}^{*}\right) s_{i}\left(1-s_{i} \frac{\lambda_{i}}{\lambda_{4}}\right)\right)\left(\int_{0}^{x_{s}^{*}} \sum_{j=1}^{3} \frac{p_{j}^{\prime}}{\lambda_{j}} e^{-\frac{\mu}{x_{j} \lambda_{j}} x} u_{j}(t, x) d x\right) \\
& +O\left(\left(|\mathbf{u}|_{H^{2}}+\left|x_{s}-x_{s}^{*}\right|\right)^{3}\right),
\end{aligned}
$$

where

$$
F(x, \mu)=\operatorname{diag}\left(\lambda_{i} p_{i} x_{i} e^{-\frac{\mu}{x_{i} \lambda_{i}} x}, i \in\{1,2,3\}\right) .
$$

We observe that, except from the last product proportional to $d$, a quadratic form in $\left(\mathbf{v}\left(x_{s}^{*}\right)^{\top}, u_{4}(0), x_{s}-\right.$ $\left.x_{s}^{*}\right)$ appears. Using successively the Young and Cauchy-Schwarz inequalities to deal with the last product, and noticing that

$$
\int_{0}^{x_{s}^{*}} e^{-\frac{\mu}{x_{i} \lambda_{i}} x} d x=\frac{\lambda_{i} x_{i}}{\mu}\left(1-e^{-\frac{\mu}{x_{i} \lambda_{i}} x_{s}^{*}}\right),
$$

we get that, for any $j \in\{1,2,3\}$,

$$
\begin{aligned}
& d\left(\sum_{i=1}^{3} u_{i}\left(x_{s}^{*}\right) s_{i}\left(1-s_{i} \frac{\lambda_{i}}{\lambda_{4}}\right)\right)\left(\int_{0}^{x_{s}^{*}} \frac{p_{j}^{\prime}}{\lambda_{j}} e^{-\frac{\mu}{x_{j} \lambda_{j}} x} u_{j}(t, x) d x\right) \leq \\
& \frac{\varepsilon_{j}}{\mu}\left(\frac{p_{j}^{\prime 2} x_{j}\left(1-e^{-\frac{\mu}{x_{j} \lambda_{j}} x_{s}^{*}}\right)}{\lambda_{j} p_{j}}\right)\left(\int_{0}^{x_{s}^{*}} p_{j} e^{-\frac{\mu}{x_{j} \lambda_{j}} x} u_{j}^{2}(t, x) d x\right)+\frac{d^{2}}{4 \varepsilon_{j}}\left(\sum_{i=1}^{3} u_{i}\left(x_{s}^{*}\right)\left(1-s_{i} \frac{\lambda_{i}}{\lambda_{4}}\right) s_{i}\right)^{2} .
\end{aligned}
$$

Using again the Cauchy-Schwarz inequality, we get that

$$
\frac{d^{2}}{4 \varepsilon_{j}}\left(\sum_{i=1}^{3} u_{i}\left(x_{s}^{*}\right)\left(1-s_{i} \frac{\lambda_{i}}{\lambda_{4}}\right) s_{i}\right)^{2} \leq \frac{d^{2}}{4 \varepsilon_{j}}\left(\sum_{i=1}^{3} u_{i}^{2}\left(x_{s}^{*}\right)\left(1-s_{i} \frac{\lambda_{i}}{\lambda_{4}}\right)^{2}\left(\sum_{j=1}^{3} e^{\frac{\mu x_{s}^{*}}{x_{i} \lambda_{i}}-\frac{\mu x_{s}^{*}}{x_{j} \lambda_{j}}}\right)\right) .
$$


Therefore, combining 67)- 70 , one has

$$
\begin{aligned}
\frac{d\left(V_{1}+V_{4}\right)}{d t} \leq & -\mu\left(V_{1}+V_{4}\right)-\mathbf{v}\left(x_{s}^{*}\right)^{\top}\left(F\left(x_{s}^{*}, \mu\right)-K^{\top} F(0, \mu) K\right. \\
& \left.-\frac{d^{2}}{4}\left(\sum_{k=1}^{3} \frac{1}{\varepsilon_{k}}\right) \operatorname{diag}\left(\left(\sum_{j=1}^{3} e^{\frac{\mu x_{s}^{*}}{x_{i} \lambda_{i}}-\frac{\mu x_{s}^{*}}{x_{j} \lambda_{j}}}\right)\left(1-s_{i} \frac{\lambda_{i}}{\lambda_{4}}\right)^{2}\right)_{i \in\{1,2,3\}}\right) \mathbf{v}\left(x_{s}^{*}\right) \\
& -\frac{x_{4} p_{4}}{\lambda_{4}} e^{-\frac{\mu}{x_{4} \lambda_{4}} x_{s}^{*}}\left(\lambda_{1} u_{1}\left(x_{s}^{*}\right)+\lambda_{2} u_{2}\left(x_{s}^{*}\right)+\lambda_{3} u_{3}\left(x_{s}^{*}\right)\right)^{2}-\lambda_{4}\left|x_{4}\right| p_{4} u_{4}^{2}(0) \\
& +\left(\mu C_{0}+\sum_{i=1}^{3}\left(x_{i} p_{i} \lambda_{i} b_{i}^{2}+x_{i} p_{i}^{\prime} b_{i}\right)\right)\left(x_{s}-x_{s}^{*}\right)^{2} \\
& +\sum_{i=1}^{3}\left(\frac{\varepsilon_{i}}{\mu}\left(\frac{p_{i}^{\prime 2} x_{i}\left(1-e^{-\frac{\mu}{\lambda_{i} x_{i}} x_{s}^{*}}\right)}{\lambda_{i} p_{i}}\right)\left(\int_{0}^{x_{s}^{*}} p_{i} e^{-\frac{\mu}{x_{i} \lambda_{i}} x} u_{i}^{2}(t, x) d x\right)\right) \\
& +\sum_{j=1}^{3}\left(2 d C_{0} s_{j}\left(1-s_{j} \frac{\lambda_{j}}{\lambda_{4}}\right)-x_{j} p_{j}^{\prime} e^{-\frac{\mu}{x_{j} \lambda_{j}} x_{s}^{*}}\right. \\
& \left.+\sum_{i=1}^{3}\left(2 x_{i} p_{i} \lambda_{i} b_{i} k_{i j}+x_{i} p_{i}^{\prime} k_{i j}\right)\right) u_{j}\left(x_{s}^{*}\right)\left(x_{s}-x_{s}^{*}\right) \\
& +O\left(\left(|\mathbf{u}|_{H^{2}}+\left|x_{s}-x_{s}^{*}\right|\right)^{3}\right) .
\end{aligned}
$$

In order to obtain an exponential decay, we first choose $\varepsilon_{i}$ such that

$$
\frac{1}{\varepsilon_{i}}=\frac{2 p_{i}^{\prime 2} x_{i}\left(1-e^{-\frac{\mu}{x_{i} \lambda_{i}} x_{s}^{*}}\right)}{\mu^{2} \lambda_{i} p_{i}}, \quad i=1,2,3 .
$$

Therefore, (71) becomes

$$
\begin{gathered}
\frac{d\left(V_{1}+V_{4}\right)}{d t} \leq-\frac{\mu}{2} V_{1}-\mu V_{4}-\mathbf{v}\left(x_{s}^{*}\right)^{\top}\left(F\left(x_{s}^{*}, \mu\right)-K^{\top} F(0, \mu) K-\frac{d^{2}}{4}\left(\sum_{k=1}^{3} \frac{1}{\varepsilon_{k}}\right) \widetilde{D}(\mu)\right) \mathbf{v}\left(x_{s}^{*}\right) \\
-\frac{x_{4} p_{4}}{\lambda_{4}} e^{-\frac{\mu}{x_{4} \lambda_{4}} x_{s}^{*}}\left(\lambda_{1} u_{1}\left(x_{s}^{*}\right)+\lambda_{2} u_{2}\left(x_{s}^{*}\right)+\lambda_{3} u_{3}\left(x_{s}^{*}\right)\right)^{2}-\lambda_{4}\left|x_{4}\right| p_{4} u_{4}^{2}(0) \\
+\left(\mu C_{0}+\sum_{i=1}^{3}\left(x_{i} p_{i} \lambda_{i} b_{i}^{2}+x_{i} p_{i}^{\prime} b_{i}\right)\right)\left(x_{s}-x_{s}^{*}\right)^{2} \\
+\sum_{j=1}^{3}\left(2 d C_{0} s_{j}\left(1-s_{j} \frac{\lambda_{j}}{\lambda_{4}}\right)-x_{j} p_{j}^{\prime} e^{-\frac{\mu}{x_{j} \lambda_{j}} x_{s}^{*}}+\sum_{i=1}^{3}\left(2 x_{i} p_{i} \lambda_{i} b_{i} k_{i j}+x_{i} p_{i}^{\prime} k_{i j}\right)\right) u_{j}\left(x_{s}^{*}\right)\left(x_{s}-x_{s}^{*}\right) \\
+O\left(\left(|\mathbf{u}|_{H^{2}}+\left|x_{s}-x_{s}^{*}\right|\right)^{3}\right)
\end{gathered}
$$

We clearly see now two terms proportional to $V_{1}$ and $V_{4}$ respectively that will bring the exponential decay, and a quadratic form in $\left(\mathbf{v}\left(x_{s}^{*}\right)^{\top}, u_{4}(0), x_{s}-x_{s}^{*}\right)$ appears. In order to simplify the quadratic form by cancelling the cross terms, we choose

$$
p_{i}^{\prime}=2 \frac{d C_{0} s_{i}\left(1-s_{i} \frac{\lambda_{i}}{\lambda_{4}}\right) e^{\frac{\mu}{x_{i} \lambda_{i}} x_{s}^{*}}}{x_{i}}, \quad i=1,2,3 .
$$

Observe that from (18), one always has $b_{i} x_{i} p_{i}^{\prime}<0$ for $i=1,2,3$, thus we can choose

$$
p_{i}=-\frac{p_{i}^{\prime}}{2 b_{i} \lambda_{i}}>0, \quad i=1,2,3 .
$$


Therefore we have, using (74), (75) and Young's inequality

$$
\begin{aligned}
\frac{d\left(V_{1}+V_{4}\right)}{d t} \leq & -\frac{\mu}{2} V_{1}-\mu V_{4}-\mathbf{v}\left(x_{s}^{*}\right)^{\top}\left(F\left(x_{s}^{*}, \mu\right)-K^{\top} F(0, \mu) K-\frac{d^{2}}{4}\left(\sum_{k=1}^{3} \frac{1}{\varepsilon_{k}}\right) \widetilde{D}(\mu)\right. \\
& \left.-\operatorname{diag}\left(\frac{3\left|x_{4}\right| p_{4} \lambda_{i}^{2}}{\lambda_{4}} e^{-\frac{\mu}{x_{4} \lambda_{4}} x_{s}^{*}}\right)_{i \in\{1,2,3\}}\right) \mathbf{v}\left(x_{s}^{*}\right) \\
& -\lambda_{4}\left|x_{4}\right| p_{4} u_{4}^{2}(0)+\left(\mu C_{0}-\frac{1}{2} \sum_{i=1}^{3}\left|x_{i} p_{i}^{\prime} b_{i}\right|\right)\left(x_{s}-x_{s}^{*}\right)^{2} \\
& +O\left(\left(|\mathbf{u}|_{H^{2}}+\left|x_{s}-x_{s}^{*}\right|\right)^{3}\right)
\end{aligned}
$$

Observe that the conditions $(18)$ and $(19)$ are satisfied for $\gamma>0$, but as the inequalities are strict, there exists $\mu>\gamma$ such that (18) and (19) are also verified with $\mu$ instead of $\gamma$. We choose such $\mu$ and using (74), one can see that

$$
\left(\mu C_{0}-\frac{1}{2} \sum_{i=1}^{3}\left|x_{i} p_{i}^{\prime} b_{i}\right|\right)<0,
$$

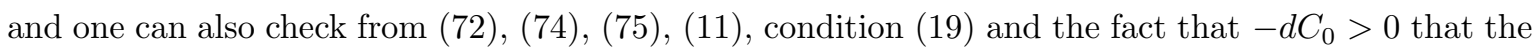
matrix defined by

$$
F\left(x_{s}^{*}, \mu\right)-K^{\top} F(0, \mu) K-\frac{d^{2}}{4}\left(\sum_{k=1}^{3} \frac{1}{\varepsilon_{k}}\right) \widetilde{D}(\mu)
$$

is positive definite. This implies that there exists $p_{4}>0$ such that the quadratic form in $\mathbf{v}\left(x_{s}^{*}\right)$ in 76 . is non-positive. Therefore

$$
\frac{d\left(V_{1}+V_{4}\right)}{d t} \leq-\frac{\mu}{2} V_{1}-\mu V_{4}+O\left(\left(|\mathbf{u}|_{H^{2}}+\left|x_{s}-x_{s}^{*}\right|\right)^{3}\right) .
$$

As $\mu>\gamma$, at least if $|\mathbf{u}|_{H^{2}}+\left|x_{s}-x_{s}^{*}\right|$ is small enough which can be guaranteed from Lemma 2.1 by requiring $\delta(\bar{T})$ small enough

$$
\frac{d\left(V_{1}+V_{4}\right)}{d t} \leq-\frac{\gamma}{2}\left(V_{1}+V_{4}\right)
$$

thus,

$$
\frac{d V}{d t} \leq-\frac{\gamma}{2} V
$$

We have derived (81) under the assumption that the trajectories of (30), 332, , 34 and (35) are of class $C^{3}$, but one can use a density argument to generalize the result for trajectories in $C^{0}\left([0, \bar{T}] ; H^{2}\left(\left(0, x_{s}^{*}\right) ; \mathbb{R}^{4}\right)\right)$ by noticing that $\gamma$ does not depend on any $C^{2}$ or $C^{3}$-norm of $\mathbf{u}$. The inequality $(81)$ is then understood in the distribution sense. One can refer to [5] or [2, Comment 4.6] for more details.

By the equivalence between the Lyapunov function $V$ and $\left(|\mathbf{u}|_{H^{2}}+\left|x_{s}-x_{s}^{*}\right|\right)^{2}$ if this last quantity is small, we get immediately the exponential stability of the null steady state of the system $[30, \sqrt{32}$, (34) and (35) for the $H^{2}$-norm with decay rate $\gamma / 4$. It remains to check that under assumption (19), 52 holds with $p_{i}^{\prime}$ and $p_{i}$ defined as (74) and (75). Indeed,

$$
\max _{i}\left(\frac{p_{i}^{\prime 2} x_{i}}{\mu \lambda_{i} p_{i}}\left(1-e^{-\frac{\mu}{x_{i} \lambda_{i}} x_{s}^{*}}\right)\right)<\frac{4 C_{0}}{3},
$$

therefore there exists $C_{0}>3 / 2$ such that the condition $(52)$ is satisfied.

So far $\delta(\bar{T})$ depends on $\bar{T}$, we next prove that for any given $T>0$, we can choose $\delta^{*}$ independent of $T$ such that (81) holds on $(0, T)$ as required in Definition 1.1

Let us now assume that $x_{s, 0} \in(0, L)$ and $\mathbf{u}_{0} \in H^{2}\left(\left(0, x_{s}^{*}\right) ; \mathbb{R}^{4}\right)$ satisfying the first order compatibility conditions and

$$
\left|\mathbf{u}_{0}\right|_{H^{2}\left(\left(0, x_{s}^{*}\right) ; \mathbb{R}^{4}\right)}+\left|x_{s, 0}-x_{s}^{*}\right|<\bar{\rho} \text { and } V\left(\mathbf{u}_{0}, x_{s, 0}\right) \leq \nu,
$$


where $\nu>0$ is going to be chosen small enough. Then, for any $t \in[0, \bar{T}]$, at least if $\nu>0$ is small enough, from (44), (53) and (81),

$$
|\mathbf{u}(t)|_{H^{2}\left(\left(0, x_{s}^{*}\right) ; \mathbb{R}^{4}\right)}+\left|x_{s}(t)-x_{s}^{*}\right|<\bar{\rho} \text { and } V\left(\mathbf{u}(t), x_{s}(t)\right) \leq \nu .
$$

Using (84) for $t=\bar{T}$ one can keep going on $[\bar{T}, 2 \bar{T}]$ and then on $[2 \bar{T}, 3 \bar{T}]$, etc. So we get that, for every $j=1,2,3, \ldots$,

$$
\begin{gathered}
V\left(\mathbf{u}(t), x_{s}(t)\right) \leq \nu, t \in[(j-1) \bar{T}, j \bar{T}], \\
\left(|\mathbf{u}(t)|_{H^{2}\left(\left(0, x_{s}^{*}\right) ; \mathbb{R}^{4}\right)}+\left|x_{s}(t)-x_{s}^{*}\right|\right)<\bar{\rho}, t \in[(j-1) \bar{T}, j \bar{T}], \\
\frac{d V}{d t} \leq-\frac{\gamma}{2} V \text { in the distribution sense on }(0, j \bar{T}) .
\end{gathered}
$$

Noticing (28), there exists a $\delta^{*}$ such that if $(13)-(14)$ hold, one has $(83)$. Thus, noticing also that for any $T>0$ there exists $j \in \mathbb{N}$ such that $(0, T) \subset(0, j T)$, one gets that the steady state $\left(\left(H^{*}, Q^{*}\right)^{\top}, x_{s}^{*}\right)$ is locally exponentially stable for the $H^{2}$-norm with decay rate $\gamma / 4$. The proof of Theorem 1.1 is thus complete.

Remark 5. Given the assumptions of Theorem 1.1, it is obvious that this stability result is robust with respect to small variations of $G$ in the feedback control. However, it is actually also robust with respect to small variations of $G_{4}$. Indeed, if $\left|G_{4}^{\prime}(0)+\lambda_{4}\right|$ is sufficiently small but with a bound independent of the state $(H, Q)^{\top}$ and $x_{s}$, we can still define $\mathcal{B}$ as in $(36)-(39)$ using the implicit function theorem. Then looking at $(54), \partial_{2} \mathcal{B}(\mathbf{0}, 0,0) \neq \mathbf{0}$, but for any $\delta>0,\left|\partial_{2} \mathcal{B}(\mathbf{0}, 0,0)\right|<\delta$ provided $\left|G_{4}^{\prime}(0)+\lambda_{4}\right|$ is sufficiently small. Then all the additional terms about $u_{4}^{2}(0)$ and $u_{i}^{2}\left(x_{s}^{*}\right)$, $i=1,2,3$ will be compensated by the fact that $p_{4}>0$ in 73 and that $\left|G_{4}^{\prime}(0)+\lambda_{4}\right|$ is sufficiently small. The rest of the proof is the same as in the case where $G_{4}^{\prime}(0)=-\lambda_{4}$.

\section{Conclusion}

In this article, we have considered the problem of the boundary feedback stabilization of an open channel with a hydraulic jump. We focused on the case where the channel has a rectangular cross section without friction or slope. The channel dynamics are modelled by a version of the homogeneous Saint-Venant equations with the water level $H$ and the flow rate $Q$ as state variables. The hydraulic jump is represented by a discontinuous shock solution of the system. The main contribution of this paper is to analyze the boundary feedback stabilization of the system with a general class of static feedback controls that require pointwise measurements of the level and the flux at the boundary and in the immediate vicinity of the hydraulic jump. In order to prove the well-posedness of the system, we first introduce a change of variables which allows to transform the Saint-Venant equations with shock wave solutions into an equivalent $4 \times 4$ quasilinear hyperbolic system which is parametrized by the jump position but has shock-free solutions. Then, by a Lyapunov approach, we show that, for the considered class of boundary feedback controls, the exponential stability in $H^{2}$-norm of the steady state can be achieved with an arbitrary decay rate and with an exponential stabilization of the desired location of the hydraulic jump. Compared with previous results in the literature for classical solutions of quasilinear hyperbolic systems, the $H^{2}$-Lyapunov function introduced in 8 (see also 2 , Section 4.4]) has to be augmented with suitable extra terms for the analysis of the stabilization of the jump position. In the case where the cross section is irregular and with friction or slope, the jump stabilization issue is much more challenging and remains an open problem.

\section{A Appendix}

In this appendix we prove that that there always exists $G$ such that $K$ and $\left(b_{1}, b_{2}, b_{3}\right)^{\top}$ defined in 11 ) satisfy (18)-(19). Let us first point out that, for every $K \in \mathbb{R}^{3 \times 3}$, there exists a linear map $G: \mathbb{R}^{4} \rightarrow \mathbb{R}^{3}$ such that the third equation of $(11)$ holds. Hence it remains only to show that there always exist $K$ and $\left(b_{1}, b_{2}, b_{3}\right)^{\top}$ satisfying $(18)$ and $(19)$. In the special case where $K=\operatorname{diag}\left(k_{i}, i \in\{1,2,3\}\right)$, the condition that the matrix defined in (19) is positive definite becomes

$$
k_{i}^{2}<e^{-\frac{\gamma}{x_{i} \lambda_{i}} x_{s}^{*}} D_{i}, \quad \forall i \in\{1,2,3\},
$$


with

$$
D_{i}:=1-\frac{2 d^{2} b_{i}}{\gamma^{2} s_{i}\left(1-s_{i} \frac{\lambda_{i}}{\lambda_{4}}\right)}\left(\sum_{k=1}^{3} b_{k} s_{k}\left(1-s_{k} \frac{\lambda_{k}}{\lambda_{4}}\right)\left(e^{\frac{\gamma x_{s}^{*}}{x_{k} \lambda_{k}}}-1\right)\right)\left(\sum_{j=1}^{3} e^{\frac{\gamma x_{s}^{*}}{x_{i} \lambda_{i}}-\frac{\gamma x_{s}^{*}}{x_{j} \lambda_{j}}}\right)\left(1-s_{i} \frac{\lambda_{i}}{\lambda_{4}}\right)^{2} .
$$

Let us look at a limiting case in 18 and take $b_{i}=-\gamma e^{-\gamma x_{s}^{*} /\left(x_{i} \lambda_{i}\right)} / 3 d s_{i}\left(1-s_{i} \frac{\lambda_{i}}{\lambda_{4}}\right)$. Then we have

$$
D_{i}=1-\frac{2}{9}\left(\sum_{k=1}^{3}\left(1-e^{-\frac{\gamma x_{s}^{*}}{x_{k} \lambda_{k}}}\right)\right)\left(\sum_{j=1}^{3} e^{-\frac{\gamma x_{s}^{*}}{x_{j} \lambda_{j}}}\right) .
$$

We denote $y=\left(\sum_{k=1}^{3} e^{-\frac{\gamma x_{s}^{*}}{x_{k} \lambda}}\right)$. Thus we get

$$
D_{i}:=1-\frac{2}{3} y+\frac{2}{9} y^{2} .
$$

This is a second order polynomial with negative discriminant, thus $D_{i}$ is always strictly positive. As $D_{i}$ depends continuously on $b_{i}$, this implies that there exist $K=\operatorname{diag}\left(k_{i}, i \in\{1,2,3\}\right)$ and $\left(b_{1}, b_{2}, b_{3}\right)^{\top}$, satisfying $(18)$ and $(19)$.

\section{Acknowledgement}

The authors would like to thank Tatsien Li and Sébastien Boyaval for their constant support. They would like also to thank National Natural Science Foundation of China (No. 11771336), ETH-ITS, ETH-FIM, ANR project Finite4SoS (No.ANR 15-CE23-0007), LIASFMA and the French Corps des IPEF for their financial support.

\section{References}

[1] Georges Bastin and Jean-Michel Coron. On boundary feedback stabilization of non-uniform linear $2 \times 2$ hyperbolic systems over a bounded interval. Systems \& Control Letters, 60(11):900906, 2011.

[2] Georges Bastin and Jean-Michel Coron. Stability and Boundary Stabilisation of 1-D Hyperbolic Systems. Number 88 in Progress in Nonlinear Differential Equations and Their Applications. Springer International, 2016.

[3] Georges Bastin and Jean-Michel Coron. A quadratic Lyapunov function for hyperbolic densityvelocity systems with nonuniform steady states. Systems \& Control Letters, 104:66-71, 2017.

[4] Georges Bastin, Jean-Michel Coron, and Brigitte d'Andréa Novel. On Lyapunov stability of linearised Saint-Venant equations for a sloping channel. Networks and Heterogeneous Media, 4(2):177-187, 2009.

[5] Georges Bastin, Jean-Michel Coron, Amaury Hayat, and Peipei Shang. Exponential boundary feedback stabilization of a shock steady state for the inviscid Burgers equation. Preprint, https://hal.archives-ouvertes.fr/hal-01723361/, 2017.

[6] Hubert Chanson. Development of the Bélanger equation and backwater equation by Jean-Baptiste Bélanger (1828). Journal of Hydraulic Engineering, 135(3):159-163, 2009.

[7] Reginald H. Clarke. The Morning Glory: An atmospheric hydraulic jump. Journal of Applied Meteorology, 11(2):304-311, 1972.

[8] Jean-Michel Coron, Georges Bastin, and Brigitte d'Andréa Novel. Dissipative boundary conditions for one-dimensional nonlinear hyperbolic systems. SIAM J. Control Optim., 47(3):1460$1498,2008$. 
[9] Jean-Michel Coron, Brigitte d'Andréa Novel, and Georges Bastin. A Lyapunov approach to control irrigation canals modeled by Saint-Venant equations. In CD-Rom Proceedings, Paper F1008-5, ECC99, Karlsruhe, Germany, pages 3178-3183, 1999.

[10] Jean-Michel Coron, Oliver Glass, and Zhiqiang Wang. Exact boundary controllability for 1-D quasilinear hyperbolic systems with a vanishing characteristic speed. SIAM J. Control Optim., 48(5):3105-3122, 2009/10.

[11] Jonathan de Halleux, Christophe Prieur, Jean-Michel Coron, Brigitte d'Andréa Novel, and Georges Bastin. Boundary feedback control in networks of open channels. Automatica, 39(8):1365-1376, 2003.

[12] Mamadou Diagne, Peipei Shang, and Zhiqiang Wang. Feedback stabilization for the mass balance equations of an extrusion process. IEEE Trans. Automat. Control, 61(3):760-765, 2016.

[13] Martin Gugat, Michael Herty, and Siegfried Müller. Coupling conditions for the transition from supersonic to subsonic fluid states. Netw. Heterog. Media, 12(3):371-380, 2017.

[14] Martin Gugat, Günter Leugering, Klaus Schittkowski, and E. J. P. Georg Schmidt. Modelling, stabilization, and control of flow in networks of open channels. In Online optimization of large scale systems, pages 251-270. Springer, Berlin, 2001.

[15] Martin Gugat and Stefan Ulbrich. The isothermal Euler equations for ideal gas with source term: product solutions, flow reversal and no blow up. J. Math. Anal. Appl., 454(1):439-452, 2017.

[16] Willi H Hager. Energy dissipators and hydraulic jump, volume 8 of Water Science and Technology Library. Springer, 1992.

[17] Amaury Hayat and Peipei Shang. A quadratic Lyapunov function for SaintVenant equations with arbitrary friction and space-varying slope. Preprint, https://hal.archives-ouvertes.fr/hal-01704710, 2017.

[18] Joachim Kuettner and Rolf F. Hertenstein. Observations of mountain induced rotors and related hypotheses: A review. In 10th Conference on Mountain Meteorology and MAP Meeting, 2002.

[19] Günter Leugering and E. J. P. Georg Schmidt. On the modelling and stabilization of flows in networks of open canals. SIAM Journal on Control and Optimization, 41(1):164-180, 2002.

[20] Ta Tsien Li and Wen Ci Yu. Boundary value problems for quasilinear hyperbolic systems. Duke University Mathematics Series, V. Duke University, Mathematics Department, Durham, NC, 1985.

[21] Vincent Perrollaz. Asymptotic stabilization of stationnary shock waves using a boundary feedback law. arXiv preprint arXiv:1801.06335, 2018. 